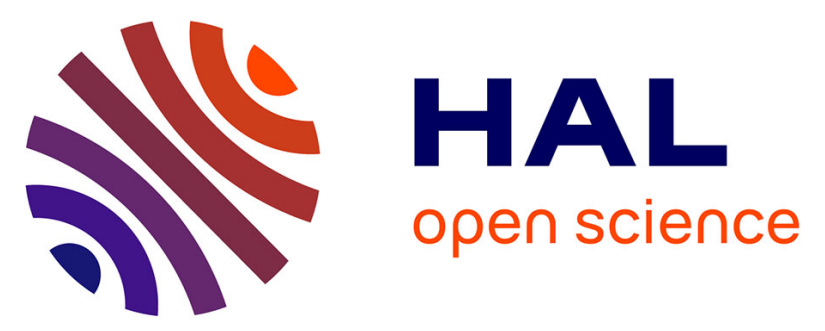

\title{
Potential of an ultraviolet, medium-footprint lidar prototype for retrieving forest structure
}

T. Allouis, S. Durrieu, P. Chazette, Jean-Stéphane Bailly, J. Cuesta, C. Véga, Pierre H. Flamant, P. Couteron

\section{- To cite this version:}

T. Allouis, S. Durrieu, P. Chazette, Jean-Stéphane Bailly, J. Cuesta, et al.. Potential of an ultraviolet, medium-footprint lidar prototype for retrieving forest structure. ISPRS Journal of Photogrammetry and Remote Sensing, 2011, 66 (6), p. S92 - p. S102. 10.1016/j.isprsjprs.2011.08.007 . hal-00654817

\section{HAL Id: hal-00654817 https://hal.science/hal-00654817}

Submitted on 23 Dec 2011

HAL is a multi-disciplinary open access archive for the deposit and dissemination of scientific research documents, whether they are published or not. The documents may come from teaching and research institutions in France or abroad, or from public or private research centers.
L'archive ouverte pluridisciplinaire HAL, est destinée au dépôt et à la diffusion de documents scientifiques de niveau recherche, publiés ou non, émanant des établissements d'enseignement et de recherche français ou étrangers, des laboratoires publics ou privés. 


\section{Potential of an ultraviolet, medium-footprint lidar proto- type for retrieving forest structure}

Tristan Allouis ${ }^{a, b},{ }^{*}$, Sylvie Durrieu ${ }^{a}$, Patrick Chazette ${ }^{c}$, Jean-

5 Stéphane Bailly ${ }^{b}$, Juan Cuesta ${ }^{d}$, Cédric Véga ${ }^{a}$, Pierre Flamant ${ }^{d}$ and Pierre Couteron ${ }^{\mathrm{e}}$

${ }^{a}$ Cemagref, UMR TETIS, 500 rue Jean-François Breton, 34093 Montpellier cedex 5, France

${ }^{b}$ AgroParisTech, UMR TETIS, 500 rue Jean-François Breton, 34093 Montpellier cedex 5, France

$10{ }^{c}$ IPSL, Laboratoire des Sciences du Climat et l'Environnement, Orme des Merisiers, 91191 Gif-sur-Yvette cedex, France

${ }^{d}$ École Polytechnique, Laboratoire de Météorologie Dynamique, 91128 Palaiseau Cedex, France

e Institut de Recherche pour le Développement, UMR AMAP, Bd de la Lironde TA A51/PS2, 34398 Montpellier cedex 5, France

15

* Corresponding author. Tel.: +334675487 19; fax: +334675487 00. E-mail address: tristan.allouis@teledetection.fr

ABSTRACT

20 The aim of the paper is to carry on methodological development for retrieving forest parameters from medium-footprint lidar signals and for assessing the performance of different sampling strategies.

The $2.4 \mathrm{~m}$ footprint lidar prototype (a profiler instrument using an ultraviolet laser) was flown above two different maritime pine stands: a young plantation (10 years old) and a

25 mature, semi-natural stand (55 years old), both in the Landes forest, France.

The vertical distribution of lidar measurements was studied for retrieving forest height parameters (mean total height, mean crown height and top height). The processing algorithm was based on an aggregation of successive signals followed by the correction of the signal attenuation along the travel through the vegetation. The performance of dif-

30 ferent sampling strategies was assessed by comparing the results for the full dataset (several fight lines over the stands) and for only a data subset (one flight line).

In addition, the horizontal distribution of height measurements was studied for identifying the planting pattern of the stands and assessing the tree spacing of the semi-natural parcel, using geostatistics.

35 We obtained a sub-metric estimation error (lidar - reference) of $0.2 \mathrm{~m}$ on the mean total height in the young stand $(-0.7 \mathrm{~m}$ in the mature stand), a bias of $-0.3 \mathrm{~m}(-0.3 \mathrm{~m})$ on the mean crown height measurement and of $0.6 \mathrm{~m}(-1.0 \mathrm{~m})$ on the top height. The 
planting pattern was also successfully identified, and the distance between trees was assessed in agreement with ground measurements.

40 Having demonstrated its ability to assess forest structure, even with a unique flight line, the lidar prototype seems to be a valuable sensor for performing fast forest inventory at regional scale. In addition, this sensor opens the way to the development of bi-functional lidar for both atmosphere and vegetation remote sensing.

45 Keywords:

Ultraviolet laser, ultra light aircraft, signal processing, attenuation, top height, crown height, plantation, semi-natural, tree spacing, semi-variogram, geostatistics

\section{Introduction}

Achieving a precise inventory of the world's forests has become a priority ever since the role that forests could play in climate regulation has been brought to light. Improved estimations of forest aboveground biomass and $\mathrm{CO}_{2}$ emissions from deforestation are needed to reduce uncertainties on the terrestrial carbon pool (ESA,

55 2008). Currently, the Forest Resource Assessment (FRA) of the United Nations Food and Agricultural Organization (FAO) is the main source of information on forest resources at the international level. However, FRA is not spatially explicit. Data are compiled at the national level and are reported through a single value of forest growing stocks, while spatially distributed estimations of growing stocks are required by

60 vegetation models. To fill this gap, remote sensing data supported by ground observations are considered the key to effective monitoring (DeFries et al., 2007). Indeed, remote sensing techniques can provide forest structure information on large areas through photogrammetry (e.g., Soenen et al., 2010), lidar (light detection and ranging) (e.g., Wynne, 2006), or both (e.g., Véga and St-Onge, 2008; Packalen et al.,

65 2009).

Lidar remote sensing not only provides surface elevation measurements as photogrammetric techniques do, but it can also provide information about vegetation structure inside and below the canopy. Even though the ground detection is still challenging in tropical or dense forests (Drake et al., 2002; Clark et al., 2004), this

70 advantage explains why many studies have focused on the use of lidar data to characterize forest environments. Tree or stand heights were successfully retrieved and the total volume and spatial organization of vegetation material were determined (e.g., Lefsky et al., 1999; Chen et al., 2007; Popescu, 2007). Due to their accuracy, forest parameters derived from lidar data can be used for forest inventory and as model inputs

75 for ecological applications (e.g., Waser et al., 2008; Silva-Santos et al., 2010). However, because lidar system parameters (i.e., laser beam divergence, laser wavelength, measurement repetition rate, waveform digitization frequency) along with the flight parameters (altitude, speed) have an impact on the recorded backscattered signals and 
the way forests are sampled (laser footprint size, distance between footprints) (Næsset, 2009), they must be chosen in relation to the scale of observation, the accuracy needed and the amount of data to be managed.

Two types of lidar systems are currently used for forest application: small- and large-footprint systems. The data from each system are processed using different techniques for retrieving forest parameters. Because of its commercial availability, small-footprint (15 to $30 \mathrm{~cm}$ ), airborne lidar systems are commonly used (e.g., Mallet and Bretar, 2009). For these systems, the range measurements between the instrument and the targets intercepted by the laser beam are extracted from multi-pulse or fullwaveform backscatter signals. The accurate three-dimensional (X, Y, Z) point cloud, which is derived from the range measurements, the scan angle and both plane attitude

90 and position, is then classically processed to create a digital terrain model (DTM) by selecting ground points among the last echoes. Then, the forest parameters are retrieved by either analyzing a Canopy Height Model (CHM) obtained by subtracting the DTM from the first echoes (e.g., Means et al., 2000; Chauve et al., 2009) or analyzing the height distribution of the points inside the point cloud at tree or plot levels (Kato et al.,

95 2009). In contrast, forest parameters can be directly computed from large-footprint lidar waveforms due to the presence of a ground return inside a 10 to $70 \mathrm{~m}$ spot. From such large-footprint airborne or satellite instruments, the stand height and total above-ground and foliage biomass can be estimated (e.g., Means et al., 1999; Lefsky et al., 2005). However, large footprint data do not allow operating at an individual tree level, and the 100 accuracy of tree height estimation suffers from the strong influence of the terrain slope. Indeed, the slope produces a mixture of vegetation and terrain echoes (Chen, 2010).

The majority of lidar systems used for forest applications use infrared or green wavelengths. However, the UV wavelength is suitable to detect the presence and the concentration of atmospheric pollutants (aerosols) (Chazette et al., 2007). Validating the

105 capacity for a UV lidar to measure 3D forest structure would then open the way to the development of bi-functional lidars able to study the interactions between vegetation and aerosols, which is particularly interesting at the interface between forest and urban areas. To that purpose, a lidar prototype was designed by the Commissariat à l'Énergie Atomique (CEA) and the Centre National de la Recherche Scientifique (CNRS) based 110 on a UV lidar sensor initially developed for atmospheric applications (Chazette et al., 2007; Raut and Chazette, 2009). The first experiments of this prototype provided measurements with a $2.4 \mathrm{~m}$ footprint (due to the laser beam divergence and a flight altitude of $300 \mathrm{~m}$ ) in order to obtain a back-scattered signal integrating information from a larger area (from sub-tree to tree level) than is observed with small-footprint lidar systems.

115 In many forest types, an increase in the footprint size would increase the probability of each laser pulse to intercept both tree tops and ground. But the drawback is a higher sensitivity of the measurement to the local topography that would limit the use of largefootprint lidar on uneven or sloped terrain. Consequently, a compromise must be reached and an intermediate footprint size could be a solution. However, new methods 
120 for processing such lidar data must be developed, taking into account that a medium footprint signal does not systematically contain a ground return, and can therefore not be processed exactly the same way as large footprint signals. On the other hand, medium footprint backscatter signals are too complex for being processed like small footprint waveforms. Indeed, the assumption made by Hofton et al. (2000) ("returning laser

125 pulse is composed of a series of potentially-overlapping reflections similar in shape to the impulse response"), would require a too large amount of signal components.

The main goal of this study is to carry on the development of methods for retrieving forest parameters that are commonly used in forestry and to confirm the poten-

130 tial of our medium-footprint system to measure forest structure. Cuesta et al. (2010) presented the first results showing the potential of the data acquired by this medium-footprint system for retrieving forest structural information on canopy top, tree crown base and undergrowth heights, through the direct process of lidar waveforms. In the current paper, an extensive analysis of both vertical and horizontal measurement

135 distributions is performed to estimate usual forest parameters from a medium footprint lidar system. Forest heights (mean total height, mean crown height and top height) are estimated by correcting the lidar signal from its attenuation through the vegetation. Planting pattern and tree spacing are retrieved by analyzing the spatial distribution of height measurements using geostatistical methods. We also evaluate the performance of

140 different sampling strategies for assessing forest parameters from a profiler system. This was done by comparing the results obtained using the full dataset or using data subsets.

\section{Material}

\section{2.1 Study area}

The experiment took place in September 2008 at the Landes forest (Landes of Gascony) in the southwest of France ( $44^{\circ} 10^{\prime} \mathrm{N}, 1^{\circ} 12^{\prime} \mathrm{W}$ ) (Fig. 1). With a planted area close to a million hectares, it is the largest maritime pine (Pinus pinaster) forest in

150 Europe and is a major economic pillar in France, with 30,000 jobs related to forestry and wood transformation. This area was also chosen because of its flat topography. Both the simplicity of the stand structure and the absence of ground slope were expected to make the process of obtaining the lidar data and interpreting the results easier.

155 For this study, we selected two stands with distinct characteristics: a young plantation (10 years old, 8 hectares) with a regular structure and high tree density along lines and a semi-natural (also called natural regeneration (Williston and Balmer, 1974)) mature stand (55 years old, 10 hectares) with a more irregular spatial distribution of trees. 


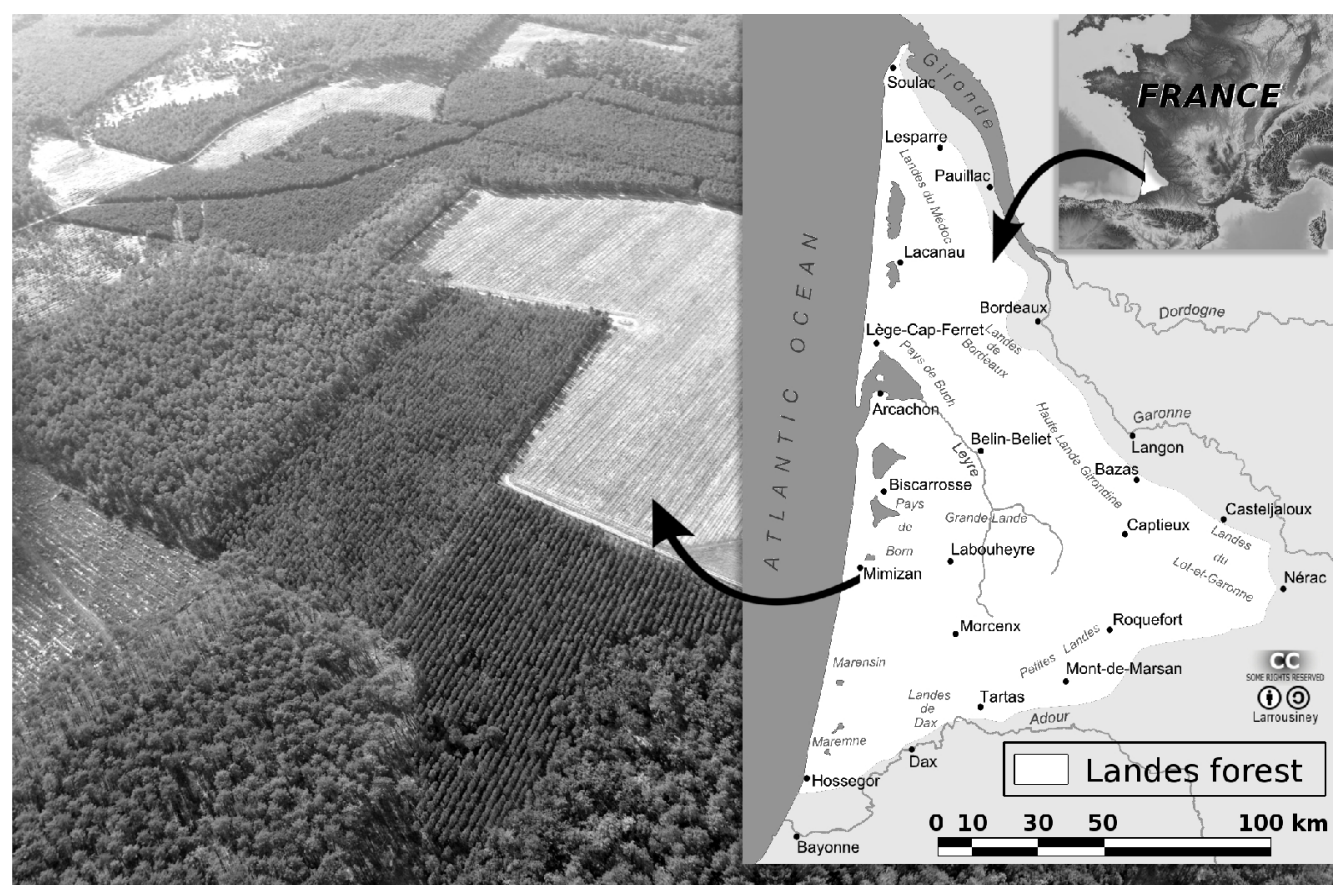

Fig. 1. Location of the study area in the Landes forest in southwestern France.

\subsection{Ultraviolet, medium-footprint lidar prototype and data set}

The lidar prototype is a Nd:YAG profiling sensor based on the LAUVA (Airborne UltraViolet Aerosol Lidar), which was initially developed by the Commissariat à l'Énergie Atomique (CEA) and the Centre National de la Recherche Scientifique (CNRS) to monitor aerosol dispersion in the low and middle troposphere (Chazette et al., 2007).

The beam divergence of the original system was set to $4 \mathrm{mrad}$, providing a $2.4 \mathrm{~m}$ footprint at a $300 \mathrm{~m}$ flight altitude. This footprint diameter was between the small- and large-footprint diameters conventionally used. The UV wavelength $(355 \mathrm{~nm})$ was retained for its ability to characterize atmospheric aerosols (Raut and Chazette, 2009) while providing adequate eye safety, since the UV radiation is absorbed by the eye

175 cornea and the crystalline before reaching the retina, even for high-energy pulses (according to the international standard IEC 60825-1; http://www.leosphere.com/). The laser emits higher energy pulses than commercial, near-infrared topographic lidars $(16 \mathrm{~mJ}$ versus $<0.2 \mathrm{~mJ})$ to offset the difference in vegetation response regarding the wavelength. The reflectance and transmittance of vegetation are approximately ten

180 times lower (Stam, 2008) in the UV wavelength than in the near-infrared wavelength traditionally used in Earth surface observation lidars. The emitted pulse duration of $5 \mathrm{~ns}$, jointly with the full-waveform receiver sampling rate of $100 \mathrm{MHz}$, lead to a vertical measurement precision close to $1.5 \mathrm{~m}$. The lidar operates at $20 \mathrm{~Hz}$ one second for every two seconds, and the data are written during the remaining second (Cuesta et al., 2010). 
The lidar was embedded on an ultra-light aircraft (ULA) to allow rapid deployment and flexibility of flight plans (see Chazette et al., 2010; Cuesta et al., 2010). Several flight lines were acquired on the study area in order to provide spatially explicit measurements on the area. The $30 \mathrm{~m} / \mathrm{s}$ ULA flight speed, the lidar operating frequency and the footprint size yielded continuous measurements along $30 \mathrm{~m}$ profiles every $30 \mathrm{~m}$

190 (Fig. 2). Due to slight ULA flight speed fluctuations, the length of the profiles varied slightly. The ULA position and attitude were given by a differential GPS (DGPS) and an electronic flight information system (EFIS). The DGPS yielded five measurements per second $(5 \mathrm{~Hz})$ of the $(\mathrm{X}, \mathrm{Y}, \mathrm{Z})$ plane position with a centimeter-level precision, while the EFIS provided one angle measurement for the first shoot of each profile of 20

195 successive shoots $(0.5 \mathrm{~Hz})$ with a $0.5^{\circ}$ accuracy. Consequently, the theoretical absolute error of the lidar profile position was about $4.5 \mathrm{~m}$, without taking into account the error in angles and position values due to the interpolation from the EFIS and DGPS measurements. However, if we estimate that the ULA movement was uniform during $1 \mathrm{~s}$, successive shoots within a 20 shoot series were positioned relatively to each others

200 with a higher accuracy $(<1 \mathrm{~m})$.

The Lidar data falling inside the two stands selected for this study were then extracted. We obtained 94 groups of 20 shoots (1,880 shoots) in the young stand and 83 groups of 20 shoots $(1,660$ shoots $)$ in the mature stand. A subset of these data was also extracted, in order to assess the ability of a unique flight line to provide tree

205 measurements that are representative of a whole stand. This subset contains 8 groups of 20 shoots (160 shoots) in each stand (Fig. 2). 


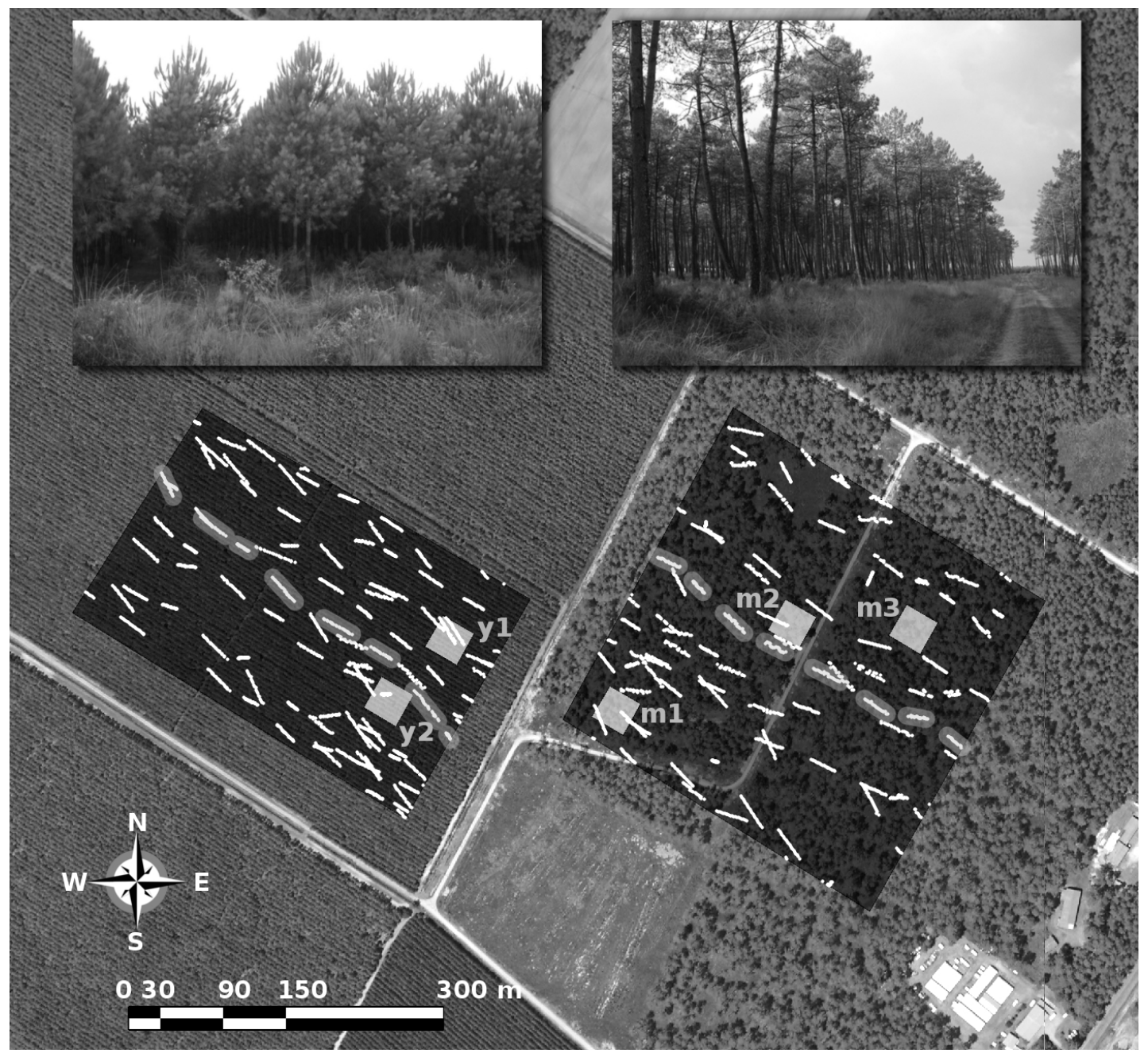

Fig. 2. The study area. The white dots represent the lidar shoots falling inside the young stand (west) and the mature stand (east). The enlighten dots (gray) represent lidar shoots of a unique flight line. The light-gray squares represent the locations of the terrain plots (young plots: y1 and $\mathrm{y} 2$. mature plots: $\mathrm{m} 1, \mathrm{~m} 2$ and $\mathrm{m} 3$ ).

\subsection{Field reference data}

Field data were collected in April 2009 on two 30x30 m plots in the young stand (planted in regular lines, plots y1, y2) and three 30x30 m plots for the mature stand (semi-natural, plots m1, m2, m3) (Fig. 2). The total height, crown base height, and crown diameter were measured for individual trees. The top heights and the distance between trees were also estimated. The field data are summarized in Table 1.

All stems were located (i.e., X, Y, Z coordinates) for each plot, and their diameter at breast height (1.30 m DBH) was measured using a tape.

The total and crown base heights were measured for all trees on the semi-natural plots using a Vertex III ultrasonic clinometer (Haglöf, Sweden). For planted plots, we 
Author-produced version of the article published in ISPRS Journal of Photogrammetry and Remote Sensing, 2011, 66(6), S92-S102. The original publication is available at http://www.isprs.org/

DOI:10.1016/j.isprsjprs.2011.08.007

225 only measured the total and crown base heights for one out of three trees for height homogeneity and because of the large number of trees.

The crown diameters were measured on the ground using a tape after positioning the maximum extend of the tree crown in two directions using a densiometer. This was done for one out of three trees along the cardinal directions (north-south and east-west)

230 in the semi-natural plots, and for one out of six trees perpendicularly and along tree lines in the planted plots.

The top height of a stand has various slightly different definitions in forestry (Nakai et al., 2010), and is often defined as the mean total height of the 100 largest (i.e. with the largest $\mathrm{DBH}$ ) trees per hectare. According to the conventional method for top

235 height estimation, the 9 largest trees in a 0.09 hectare plot must be selected in the field (Garcia and Batho, 2005). Because the lidar cannot directly measure DBH, the reference top height of a stand is defined in this paper as the mean total height of the 9 tallest trees in each plot.

The reference tree spacing was simply computed as the mean of the nearest

240 neighbor distance of each tree in the plot. Because the plantations had different tree spacing along the lines and between the lines, the tree spacing trees was not used in this study for plantations.

\section{Table 1}

245 Plot characteristics. Direction A is along plantation lines for young plots and north for mature plots. Direction B is between lines for young plots and east for mature plots.

\begin{tabular}{|c|c|c|c|c|c|}
\hline & $\begin{array}{l}\text { Plot y1 } \\
\text { (young) }\end{array}$ & $\begin{array}{l}\text { Plot y2 } \\
\text { (young) }\end{array}$ & $\begin{array}{l}\text { Plot m1 } \\
\text { (mature) }\end{array}$ & $\begin{array}{l}\text { Plot m2 } \\
\text { (mature) }\end{array}$ & $\begin{array}{l}\text { Plot m3 } \\
\text { (mature) }\end{array}$ \\
\hline \multirow{2}{*}{ Number of trees } & 127 & 102 & 25 & 15 & 17 \\
\hline & \multicolumn{2}{|c|}{229} & \multicolumn{3}{|c|}{57} \\
\hline \multirow{2}{*}{$\begin{array}{c}\text { Mean total height } \\
\pm \text { standard deviation }(\mathrm{m})\end{array}$} & $\begin{array}{c}9.5 \\
\pm 0.9\end{array}$ & $\begin{array}{c}9.1 \\
\pm 0.8\end{array}$ & $\begin{array}{c}20 \\
\pm 1.6\end{array}$ & $\begin{array}{r}21.9 \\
\pm 0.9\end{array}$ & $\begin{array}{c}23 \\
\pm 2.3\end{array}$ \\
\hline & \multicolumn{2}{|c|}{$9.3 \pm 0.9$} & \multicolumn{3}{|c|}{$21.6 \pm 2$} \\
\hline \multirow{2}{*}{$\begin{array}{l}\text { Mean crown base height } \\
\pm \text { standard deviation }(\mathrm{m})\end{array}$} & $\begin{array}{c}4.5 \\
\pm 0.8\end{array}$ & $\begin{array}{c}5.3 \\
\pm 0.5\end{array}$ & $\begin{array}{c}14 \\
\pm 1.5\end{array}$ & $\begin{array}{r}15.6 \\
\pm 1.1\end{array}$ & $\begin{array}{r}16.3 \\
\pm 1.3\end{array}$ \\
\hline & \multicolumn{2}{|c|}{$4.9 \pm 0.8$} & \multicolumn{3}{|c|}{$15.3 \pm 1.6$} \\
\hline \multirow{2}{*}{$\begin{array}{l}\text { Crown dimensions in } \\
\text { direction } \mathrm{A}(\mathrm{m})\end{array}$} & 2.7 & 2.8 & 6.6 & 5.5 & 8.6 \\
\hline & \multicolumn{2}{|c|}{2.8} & \multicolumn{3}{|c|}{7.0} \\
\hline \multirow{2}{*}{$\begin{array}{l}\text { Crown dimensions in } \\
\text { direction } B(m)\end{array}$} & 3.4 & 3.1 & 6.0 & 5.1 & 7.2 \\
\hline & \multicolumn{2}{|c|}{3.3} & \multicolumn{3}{|c|}{6.1} \\
\hline $\begin{array}{c}\text { Top height } \\
\pm \text { standard deviation }(\mathrm{m})\end{array}$ & $\begin{array}{c}10.1 \\
\pm 0.6\end{array}$ & $\begin{array}{c}9.6 \\
\pm 0.4\end{array}$ & $\begin{array}{r}22.3 \\
\pm 1.1\end{array}$ & $\begin{array}{l}22.5 \\
\pm 0.6\end{array}$ & $\begin{array}{r}24.8 \\
\pm 1.5\end{array}$ \\
\hline
\end{tabular}




\begin{tabular}{|c|c|c|}
\hline & $9.9 \pm 0.5$ & $23.2 \pm 1.5$ \\
\hline Tree spacing $(\mathrm{m})$ & $N A$ & 5.1 \\
\hline
\end{tabular}

\section{Method}

The backscattered signal of our system was not composed of a succession of narrow and separated echoes, nor did it systematically contain both tree and ground returns in the same waveform, which is essential for the direct extraction of tree heights from the individual waveform. Consequently, we developed specific methods to process the medium-footprint lidar waveforms based on a three-phase approach. The first phase

255 (described in section 3.1) consisted of aggregating the 20 successive waveforms corresponding to a pulse emission sequence of one second. A logarithmic function was then used to correct the waveform sum from the laser attenuation inside the canopy and to retrieve ground position, mean total and mean crown height on a $30 \mathrm{~m}$-long transect (Fig. 3). This methodology reduces ambiguity in the detection of ground and tree

260 structural parameters due to the foliage occlusion effect, which was noted in Cuesta et al. (2010). In the second phase (described in section 3.2), individual waveforms were corrected and individual total heights were retrieved even for waveforms without a ground return, by considering the ground position estimated in phase one. This second phase detects tree heights at a finer scale to compute stand top heights. In the third

265 phase (described in section 3.3), geostatistics were used to identify the planting pattern of the stands and estimate tree spacing from individual height measurements. The results were then compared to field data to quantify the accuracy of the lidar-derived parameters, considering that field plot measurements are statistically representative of the whole stands. The three phases were applied on the whole dataset, and phase 1 and phase 2 were also applied on the one flight line subset. 


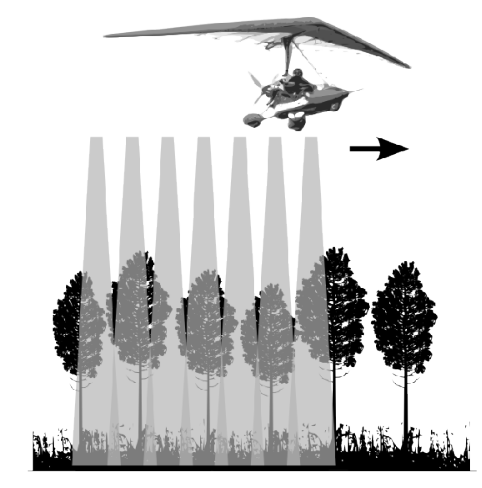

PHASE 1

- Sum of the 20 successive waveforms

- Correction of signal attenuation

- Ground position retrieval

- Mean total height and mean crown base

height extraction

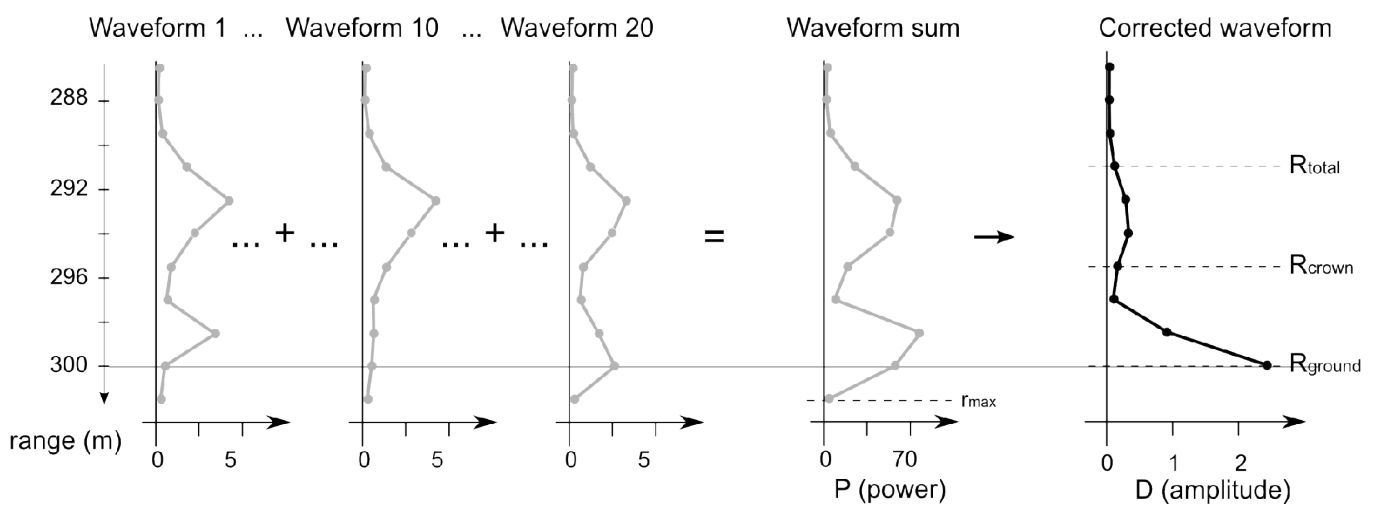

Fig. 3. Overview of the algorithm's first phase, for the extraction of mean total height and mean crown base height (phase 1).

\subsection{Waveform aggregation and signal attenuation correction}

To retrieve the total tree height, we needed to have both a return from the canopy top and a return from the ground in a lidar waveform. Because the individual waveforms, which corresponded to a signal backscattered by a $4.5 \mathrm{~m}^{2}$ circular area, did

280 not systematically include a ground return, we chose to aggregate the 20 successive shoots. This approach allowed us to increase the probability of obtaining a ground return in the waveform sum corresponding to a signal backscattered in an area of about $30 \mathrm{~m} \times 2.4 \mathrm{~m}\left(72 \mathrm{~m}^{2}\right)$. This method also increased the signal-to-noise ratio within the square root of the amount of summed waveforms, i.e., $\quad \sqrt{20} \approx 4.5 \quad$ (Measures, 1984),

285 because the noise can be approximated as a random process.

Before summing, each of the 20 successive waveforms was first georeferenced, and their altitudes were synchronized using the plane attitude information (DGPS + IMU). The amplitudes of the 20 successive waveforms were then summed. To prepare the correction of the laser attenuation, an estimation of the last echo location $\left(r_{\max }\right)$ was

290 performed by selecting the last point of the waveform sum exceeding twice the maximum noise level. This maximum noise level corresponded to the maximum waveform amplitude during the laser pulse propagation through the atmosphere. 
Although it may increase the probability of a non-detection, this threshold level was set using a generate and test method to decrease the risk of false alarm (the probability of which can be considered equal to 0 ).

The waveform sums were then corrected to account for the attenuation of the laser beam intensity along its passage through the canopy. To this aim, we adapted the MacArthur and Horn equation used for computing foliage profiles (MacArthur and Horn, 1969). A similar approach was used by Lefsky et al. (1999) to compute canopy

300 height profiles. Our adapted equation corrected the signal attenuation inside the vegetation using a logarithmic function:

$$
D_{r_{i}}=\ln \left(\frac{\sum_{r_{i}}^{r_{\max }} P(r)}{\sum_{r_{i+1}}^{r_{\max }} P(r)}\right)
$$

Where is the amplitude of the corrected waveform at range $r_{i}$ from the plane with $i \in\left[1, r_{\max -1}\right]$, and $P_{r} \mid \quad$ is the recorded power as a function of range. The correction is

305 calculated for each interval between two successive ranges from the atmosphere to $r_{\max }$ (Fig. 3).

Contrary to the method for processing ground measurements proposed by MacArthur and Horn (1969), we performed the correction calculation from sky to

310 ground to deal with the specificity of airborne lidar measurements. As a result, our corrected waveform contained a component of energy reflected from the ground.

Because we did not take into account the difference in reflectance between ground and vegetation, we could not call the corrected waveforms "foliage profiles". However, this correction allowed us to obtain an amplitude profile close to a real foliage profile and to

315 have an enhanced ground peak, making the determination of the ground location easier and more accurate (Fig. 3).

\subsubsection{Mean total height and mean crown height assessment}

The last peak location of the corrected waveform was considered to correspond

320 to the ground range ( $\mathrm{R}_{\text {ground }}$ ) (Fig. 3). The first point of the corrected waveform that exceeded the noise threshold was considered to correspond to the top of canopy range $\left(\mathrm{R}_{\text {total }}\right)$. This noise threshold was set as twice the noise level measured on the corrected waveform in the atmosphere using a generate and test method to support non-detection instead of false alarm. The point located after the strongest decrease in the corrected

325 waveform and a three-meter minimum above $\mathrm{R}_{\text {ground }}$ (to avoid undergrowth detection) was considered to correspond to the crown base range $\left(R_{\text {crown }}\right)$. The distance between $\mathrm{R}_{\text {ground }}$ and $\mathrm{R}_{\text {total }}$ was assumed to be the mean total height of the trees located inside the 20 lidar footprints used for computing the corrected waveform. The distance between 
$\mathrm{R}_{\text {ground }}$ and $\mathrm{R}_{\text {crown }}$ was assumed to be the mean crown base height. We calculated the mean total height and the mean crown base height of all 20 shoot sequences included in the studied stands. Heights were also calculated for shoot sequences of a unique flight line. The resulting values were analyzed against the field-based measurements.

\subsection{Re-processing of individual waveforms}

The first phase was designed to provide robust measurements of the ground position and mean tree height. However, finer-scale measurements were necessary to obtain information about the spatial variability of the stands' structural characteristics.

Hence, we corrected the lidar signal as presented in section 3.1 but now for each

340 individual waveform instead of the waveform sum. The process was performed from the atmosphere to the $\mathrm{r}_{\max }$ determined in the first phase for each group of 20 shoots. For each individual corrected waveform, we extracted the total height range $\mathrm{R}_{\text {IndivTop }}$ but kept the robust measurement of $R_{\text {ground }}$ extracted in the first phase. The individual total heights were then calculated as the difference between $R_{\text {IndivTop }}$ and $R_{\text {ground. }}$.

\subsubsection{Top height assessment}

To assess the lidar top height, the tallest individual height measurement in each series of 20 shoots was first selected. To keep the ratio of 100 trees measured per hectare specified in the definition of the dominant height in forestry, we selected

350 approximately $72 \%$ of the tallest individual measurements while considering a theoretical measurement area of 0.072 hectares for the 20 shoot lidar footprint ( $30 \mathrm{~m} \mathrm{x}$ $2.4 \mathrm{~m}$ ). Top height was calculated for both the entire dataset and for shoot sequences of a unique flight line. The resulting values were compared to the reference top height measurements.

\subsection{Semi-variogram computation from individual measurements}

Individual measurements of total height provided additional precision for studying the spatial variation of forest stands. In this section, we present geostatistical-

360 based methods for assessing planting patterns and tree spacing. The following methods were applied only on the whole dataset.

\subsubsection{Semi-variograms and their use in remote sensing}

High spatial resolution sensors allow the textures of observed surfaces to be

365 identified. For targets having a periodic pattern, the best way to identify texture is to use the Fourier transform (e.g., Delenne et al., 2008) or wavelet transform (e.g., Ranchin et al., 2008). However, these techniques can only be applied to data resulting from regularly spaced measurements, such as measurements provided by image sensors. Because our lidar measurements were irregularly distributed in space, the Fourier or 
370 wavelet transforms could not be performed. Consequently, we used variograms, which can be applied to regular or irregular data as well as continuous or discontinuous data.

In spatial statistics, the variogram, or semi-variogram, is a function describing the spatial correlation in observations measured at sample locations. The variogram displays the variance within groups of observations plotted as a function of distance

375 between the observations. For all observations : at locations $s_{i, \ldots, s_{k}}$ with $i=1, \ldots, k$, the omnidirectional variogram $\hat{\gamma}(d) \quad$ is defined as (Cressie, 1993):

$$
\hat{\gamma}(d)=\frac{1}{2|N(d)|} \sum_{(i, j) \in N(d)}\left(z_{i}-z_{j}\right)^{2}
$$

Where $d$ is the distance between observations, $N(d)$ denotes the set of distinct pairs of observations $\quad(i, j) \quad$ such that $\quad\left|s_{i}-s_{j}\right|=d \pm \Delta d \quad(\Delta d$ is a tolerance distance) and $|N(d)|$ is

380 the number of distinct pairs in the set.

The variogram can also be computed with pairs of observations satisfying a directional constraint (directional variogram), such as

$$
\hat{\boldsymbol{\gamma}}(d, \boldsymbol{\theta})=\frac{1}{2|N(d, \boldsymbol{\theta})|} \sum_{(i, j) \in N(d, \boldsymbol{\theta})}\left(z_{i}-z_{j}\right)^{2}
$$

Where $N(d, \theta) \quad$ denotes the set of distinct pairs of observations $\quad[i, j$ such that

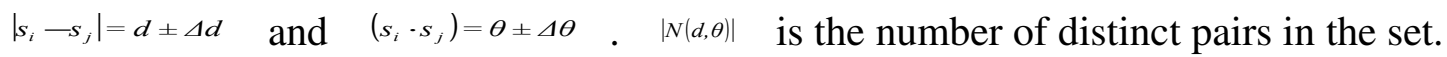

A variogram plotted from observations is called an empirical variogram, and it can be approximated by a model function to estimate the following three parameters: nugget, sill and range. The nugget represents the semi-variogram discontinuity at the origin (which shows the spatially random component), the sill is the maximum variance

390 when the distance tends toward infinity and the range is the distance at which the variogram reaches the sill.

Semi-variograms have been used in remote sensing for textural information assessment, but they were applied to optical images (St-Onge and Cavayas, 1997). Consequently, the presence of a planting pattern was indirectly studied though sun-

395 induced shadows on the image. In contrast, lidar data give pure elevation information that can be directly used to study the spatial structure of a stand. A recent study used variograms to initialize the size of a search window used for locating the apex of trees (Tesfamichael et al., 2009). In our case, the variograms were computed with a $2.4 \mathrm{~m}$ binning to make them consistent with the laser footprint size. Because of the footprint

400 size and the sampling pattern of the lidar measurements, it was not possible to precisely assess the stand structure. However, we assumed that the identification of a directional structure tendency in a stand and the mean tree spacing could be derived from semivariograms.

\subsubsection{Planting pattern assessment}

Tree position and the spatial distribution of tree heights can present various patterns according to the origin of the stand, its age and the management practices. In 
mono-specific even-aged stands, two main patterns are commonly found according to the origin of the stand: planted or semi-natural. Unlike a semi-natural stand, a plantation

410 can present a strong directional pattern. This pattern is studied in this paper through the computation of directional variograms.

Considering all pairs of total height measurements, the empirical variograms were then computed for six directions from the north and clockwise $\left(0^{\circ}, 30^{\circ}, 60^{\circ}, 90^{\circ}\right.$, $120^{\circ}, 150^{\circ}$ ) with a tolerance angle of $15^{\circ}$. This range of angles was chosen to explore

415 anisotropy with a better accuracy than using the four cardinal directions alone, while keeping a sufficient number of observation pairs $(>80)$ in each variogram sample. The existence of a directional anisotropy was studied in each stand through visual interpretation of the directional variogram shapes. A difference in shape according to different directions was expected for stands planted in lines due to the distance between trees along the line being shorter than the inter-line distance.

\subsubsection{Tree spacing assessment}

When no clear anisotropy was revealed by the previous analysis with empirical directional variograms, the omnidirectional variogram was computed and modeled.

A canopy variogram is traditionally approximated with widely accepted circular or spherical models (Curran, 1988; St-Onge and Cavayas, 1997; Tesfamichael et al., 2009) with ranges that provide a good estimation of the mean spacing between objects (see Wackernagel, 1995, p.45). However, we assumed that the spatial distribution of trees in semi-natural stands could be modeled using a random process called the "dead

430 leaves model" (Lantuejoul 2002, p.175). This model is "constructed from hard spheres of constant diameter" (Gille, 2002) that represent tree crowns. This model was also demonstrated to be suitable for describing the spatial correlation in a pine stand and was used by Boone and Bullock (2008) to characterize inter-tree competition. Because Gille (2002) showed that the outcome of this random process is a variogram that can be

435 approximated by a Matern model, we used this model to approximate the empirical variogram on the semi-natural stand. The Matern model generalizes exponential models with asymptotic sill. For such models, the "practical range" (which is equivalent to the range parameter of fixed sill models) corresponds to the distance where the semi variance reaches $95 \%$ of the sill. It equals three times the range parameter given in the equation (4) (Wackernagel, 1995, p.41). Consequently, the practical range of the Matern model is used to assess the mean spacing between objects. The Matern model equation is (Ribeiro and Diggle, 2010):

$$
C(d)=\frac{1}{\Gamma(v) \times 2^{v-1}} \times\left(\frac{d}{\varphi}\right)^{v} \times K_{v}\left(\frac{d}{\varphi}\right)
$$

445 Where $\varphi$ is the range parameter, $v$ is the smoothness parameter, $\mathrm{K} v$ is the modified Bessel function of the third kind of order $v$ and $\Gamma$ is the gamma function. The function is valid for $\varphi>0$ and $v>0$. 
The Matern model was then automatically fitted to the empirical variogram to adjust its parameters (nugget, sill and range) using the $\mathrm{R}$ software with the geoR package (Ribeiro and Diggle, 2010). This software provides a non-linear least squares adjustment algorithm weighted by the number of pairs in each distance class. Afterward, the practical range of the fitted model was computed as an estimation of the mean spacing between trees.

\section{Results}

\subsection{Mean total height and mean crown height assessment}

Table 2 shows a quantitative assessment of the mean total and mean crown heights. There is a slight overestimation of the mean total height in the young stand and a slight underestimation in the mature stand. Nevertheless, the absolute values of these errors are lower than the standard deviation of the reference tree heights $(0.9 \mathrm{~m}$ in the young stand and $2 \mathrm{~m}$ in the mature stand; see Table 1).

465 The error on the mean crown height presents a slight underestimation, but its absolute value is also lower than the standard deviation of the reference crown heights ( $0.8 \mathrm{~m}$ in the young stand and $1.6 \mathrm{~m}$ in the mature stand; see Table 1$)$.

The values computed for the entire stand and for one flight line are consistent and differ from only $10 \mathrm{~cm}$ to $20 \mathrm{~cm}$.

\section{Table 2}

Tree (total and crown) heights extracted from corrected waveform sums and their comparison with mean heights calculated from field measurements. $\mathrm{n}$ is number of measurements (corrected waveform sum) for each stand.

\begin{tabular}{|c|c|c|c|c|}
\hline & \multicolumn{2}{|c|}{ Young plantation } & \multicolumn{2}{c|}{ Mature stand } \\
\cline { 2 - 5 } & $\begin{array}{c}\text { Whole data set } \\
(\mathrm{n}=94)\end{array}$ & $\begin{array}{c}\text { One flight line } \\
(\mathrm{n}=8)\end{array}$ & $\begin{array}{c}\text { Whole data set } \\
(\mathrm{n}=83)\end{array}$ & $\begin{array}{c}\text { One flight line } \\
(\mathrm{n}=8)\end{array}$ \\
\hline $\begin{array}{c}\text { Mean of lidar total heights } \\
\pm \text { standard deviation (m) }\end{array}$ & $\begin{array}{c}9.5 \\
\pm 1.1\end{array}$ & $\begin{array}{c}9.6 \\
\pm 1.6\end{array}$ & $\begin{array}{c}20.9 \\
\pm 1.6\end{array}$ & $\begin{array}{c}21 \\
\pm 1.3\end{array}$ \\
\hline $\begin{array}{c}\text { Mean total height bias } \\
\text { (lidar - field) }(\mathrm{m})\end{array}$ & 0.2 & 0.3 & -0.7 & -0.6 \\
\hline $\begin{array}{c}\text { Mean of lidar crown base } \\
\text { heights }\end{array}$ & 4.6 & 4.7 & 15.0 & 15.2 \\
\pm standard deviation (m) & \pm 1.2 & \pm 1.5 & \pm 1.9 & \pm 1.5 \\
\hline $\begin{array}{c}\text { Mean crown base height bias } \\
\text { (lidar - field) (m) }\end{array}$ & -0.3 & -0.2 & -0.3 & -0.1 \\
\hline
\end{tabular}




\subsection{Top height assessment}

$72 \%$ of the tallest individual measurements represent 59 selected spots for the young stand and 68 for the mature stand, when using the whole data set. When considering only one flight line, 6 measurements were used to compute top height. The top height was estimated with a $1 \mathrm{~m}$ underestimation within the mature stand (Table 3) but this assessment was not significantly different from the reference measurement because the absolute error was lower than the standard deviation of the reference measurements. In contrast, the top height derived from the lidar data in the

485 young plantation was overestimated by $0.60 \mathrm{~m}$, which is $0.1 \mathrm{~m}$ above the standard deviation of the reference measurements.

The values computed for the entire stand and for one flight line are consistent and differ from only $0 \mathrm{~cm}$ to $20 \mathrm{~cm}$.

\section{Table 3}

Top heights extracted from corrected waveforms and their comparison with those calculated from field measurements. $\mathrm{n}$ represents the number of measurements used to compute the top height.

\begin{tabular}{|c|c|c|c|c|}
\hline \multirow{2}{*}{} & \multicolumn{2}{|c|}{ Young plantation } & \multicolumn{2}{c|}{ Mature stand } \\
\cline { 2 - 5 } & $\begin{array}{c}\text { Whole data } \\
\text { set (n=59) }\end{array}$ & $\begin{array}{c}\text { One flight } \\
\text { line (n=6) }\end{array}$ & $\begin{array}{c}\text { Whole data } \\
\text { set (n=68) }\end{array}$ & $\begin{array}{c}\text { One flight } \\
\text { line (n=6) }\end{array}$ \\
\hline Lidar top height & 10.5 & 10.5 & 22.2 & 22 \\
\pm standard deviation (m) & \pm 1.2 & \pm 1.3 & \pm 1.1 & \pm 1.2 \\
\hline Top height bias (lidar - field) (m) & 0.6 & 0.6 & -1.0 & -1.2 \\
\hline
\end{tabular}

\subsection{Planting pattern assessment}

Directional variograms are shown in Figs. 4 and 5 for the young and mature stands, respectively. Fig. 4 shows a significant nugget anisotropy (Zimmerman, 1993). A directional anisotropy can be seen for a distance between measurements ranging between 0 and $4 \mathrm{~m}$, through two distinct clusters of semi-variance values for the first distance class $(1.2 \mathrm{~m})$. One cluster is composed of $0^{\circ}$ to $60^{\circ}$ directions without any spatial correlation (stationary or decreasing semi-variance along distance), while the other contains directions from $90^{\circ}$ to $150^{\circ}$ with little spatial correlation (increasing semi-variance) for distances up to $4 \mathrm{~m}$.

Because the directions of the plantation lines were close to $120^{\circ}$ (see Fig. 2), the first cluster refers to the perpendicular direction of the lines, while the second cluster refers to the same direction as the plantation lines. The presence of clusters can be explained by a stronger variance of lidar-based vegetation heights in the perpendicular direction of the plantation lines than along the flight lines. In the perpendicular 
510 direction, the height measurements alternatively reached the tree tops and the ground/low vegetation due to gaps between lines. More homogeneous measurements were made along the lines due to the absence of gaps between tree crowns.

In contrast, Fig. 5 does not show semi-variance clusters in specific directions. Thus, the mature stand did not show a specific planting pattern, and it was consequently 515 assumed not to be planted in lines. However, the gradient of variance depending on variogram direction shows a geometric anisotropy between 0 and $4 \mathrm{~m}$. This observation can be explained by the crown shape anisotropy. Actually, the mean crown dimensions are $0.9 \mathrm{~m}$ greater in the north direction than the east direction, as shown by the reference data in Table 1, which typically produces a geometric anisotropy.

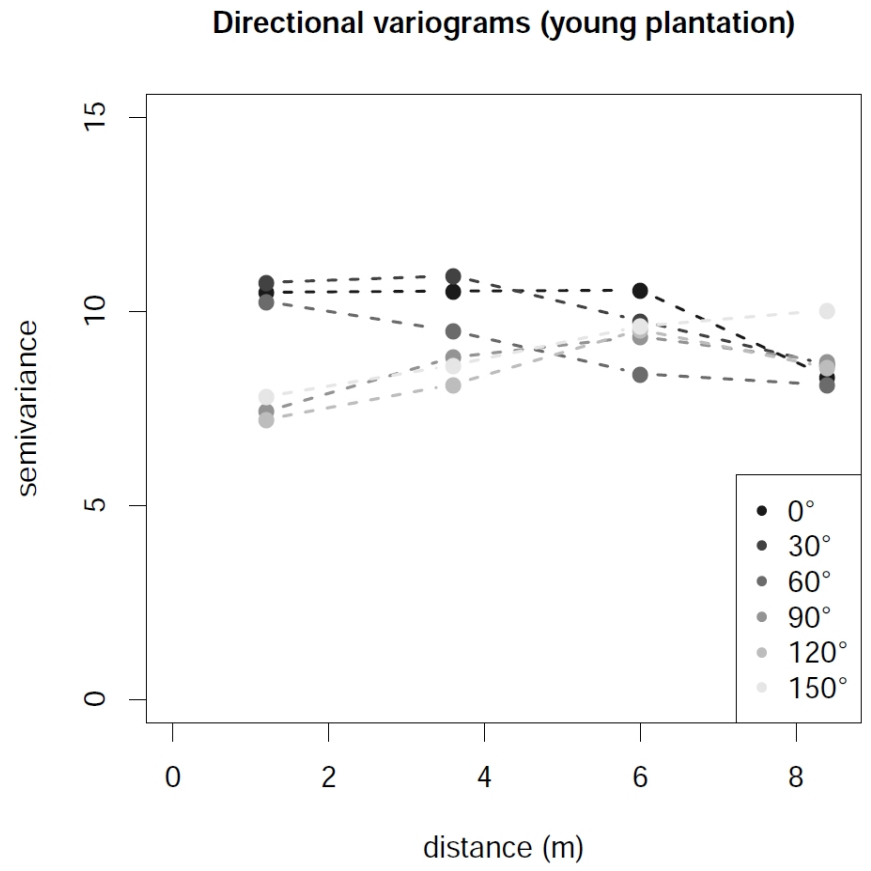

Fig. 4. Directional variograms for six directions in the young plantation. 
Author-produced version of the article published in ISPRS Journal of Photogrammetry and Remote Sensing, 2011, 66(6), S92-S102.

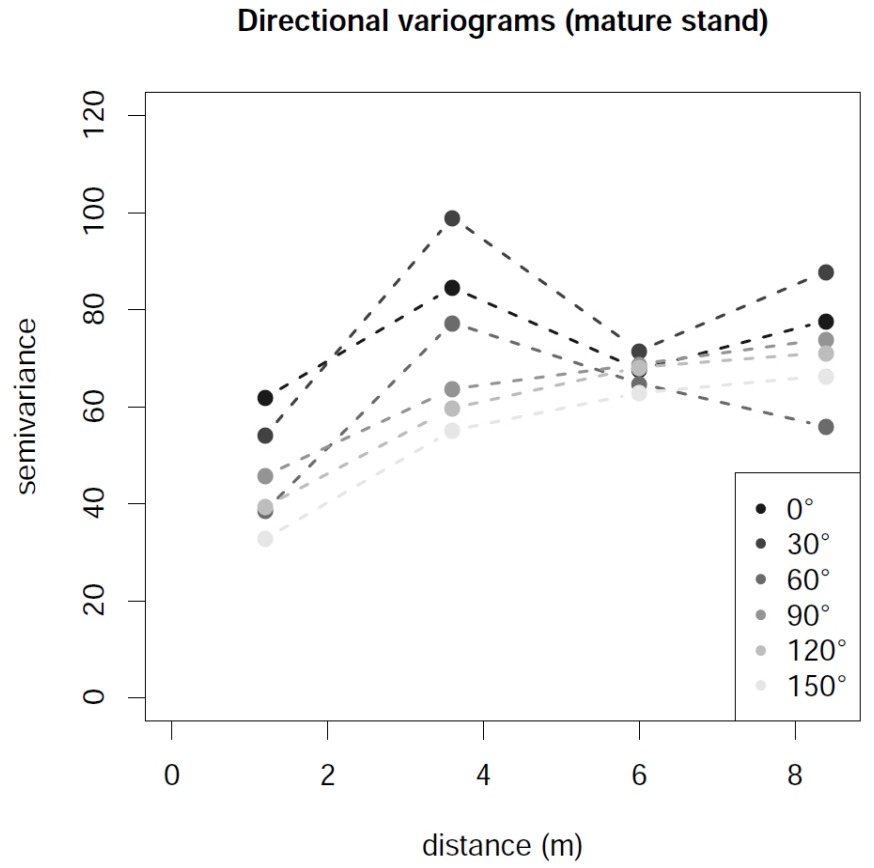

525

Fig. 5. Directional variograms for six directions in the mature semi-natural stand.

\subsection{Tree spacing assessment}

Fig. 6 shows the results of the Matern model fitted on the empirical variogram

530 for the mature stand. The "practical range" of the variogram gave an estimation of the mean spacing between trees of $5.1 \mathrm{~m}$ ( $3 \times 1.7)$, which has exactly the same value as the estimation derived from the field measurements. 


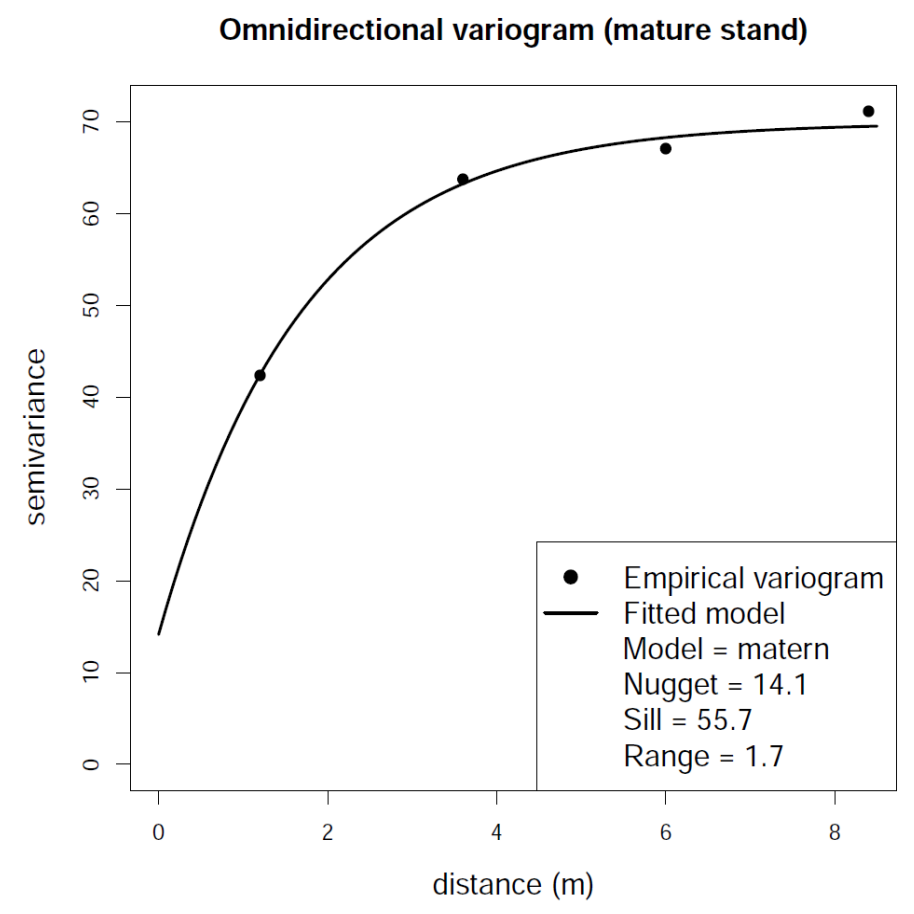

Fig. 6. Omnidirectional variogram for the mature, semi-natural stand.

\section{Discussion}

Methods presented in this paper were successfully tested on even-aged,

540 monospecific stands. If inaccurate crown base height detection is liable to occur in multi-layered forests, retrieval of top height aims to work on most forests. The only requirement is the forest to be transparent enough for the lidar signal to hit the ground and come back. But the method presented here to extract heights was designed to avoid problems linked to non-systematic ground detection. Moreover, the number of lidar

545 shoots to aggregate can be tuned according to the probability of a shoot to hit the ground. A denser forest will need a larger number of shoots to be summed for retrieving local ground position.

\subsection{Global positioning and terrain influence}

As explained in the section 2.2, the global positioning accuracy of laser profiles (series of 20 lasers shoots) was around $4.5 \mathrm{~m}$, with a relative accuracy lower than $1 \mathrm{~m}$ between spots of a same series. This is a clear restriction for computing high resolution digital terrain models. However, methods proposed in this paper for measuring trees and

555 planting patterns were designed to get rid of this inaccurate positioning.

To this aim, tree heights were measured relatively to the ground position detected inside an aggregated waveform, whereas for small-footprint lidar data heights are extracted 
after an absolute positioning of each echo. This relative measurements of tree height allowed us to accurately measure trees.

560 However, height measurements could not be accurately positioned in a global referential. Consequently, a tree to tree comparison with reference measurement could not be performed as it is often done in studies using small-footprint data. Instead, the comparison was statistically performed at stand level, considering that the reference plots were representative of the entire stands. Despite the low absolute positioning

565 accuracy, we were able to recognize the planting pattern of stands and estimate the distance between trees. The reason is that variograms were computed between 0 and $8 \mathrm{~m}$. At these distances, the majority of measurements pairs came from the same series of shoots and they consequently benefit from the sub metric relative positioning accuracy.

Retrieving canopy top and ground position from the same waveform seems similar to direct methods used for large-footprint data (Chen, 2010), but it is here much less sensitive to terrain slope influence on ground positioning, thanks to a smaller footprint size. Considering a $2.4 \mathrm{~m}$ footprint containing a ground echo, the inaccuracy

575 of ground positioning will lead to a $2.4 \mathrm{~m}$ error in a $100 \%$ terrain slope. In the case of a waveform aggregation the error will rise, according to a bigger footprint size, but reprocessing individual waveforms (which is not possible with large-footprint data) for refining the ground positioning is conceivable and would lead to improve height estimates.

\subsection{Phase 1 and 2: Mean total height, mean crown height, and top height assessment}

The mean total height assessment did not seem to notably benefit from the correction of the signal attenuation compared to Cuesta et al. (2010). However, the

585 accuracy estimation of the mean crown height was increased by about $1.7 \mathrm{~m}$ compared to the previous study. This result demonstrates the importance of correcting the signal from its attenuation when retrieving structural parameters inside the canopy.

The lidar measurement was expected to underestimate the tree height due to the combination of two factors. First, the resolution $r$ of the waveform digitizer $(1.5 \mathrm{~m})$ should lead to a mean underestimation of the tree height between $\mathrm{r} / 2$ and $\mathrm{r}(0.75 \mathrm{~m}$ to $1.5 \mathrm{~m})$, which is partially compensated for by the large pulse width $(5 \mathrm{~ns}$, i.e., $1.5 \mathrm{~m})$ of the current system. Second, the tree heights were underestimated due to a reduced probability of the laser to exactly reach the tree tops (e.g., Chauve et al., 2009). Indeed, the tree tops are highly punctuate regarding the stand area, especially in mature stands, and the probability of detecting them is inversely proportional to the number of trees. The results found were in agreement with these statements, as we observed an underestimation between $0.1 \mathrm{~m}$ and $0.7 \mathrm{~m}$ for almost retrieved heights. Nevertheless, 
the total height estimation for the young plantation was overestimated. A total height underestimation of tall trees jointly with an overestimation of young trees was already reported by Brandtberg et al. (2003). The explanation they gave was that field measured tree heights were affected by error introduced by the field personnel. But we had no difficulties to locate top of trees from the ground in our young plantation. A possible explanation lies in the consequence of the vertical sampling of the backscatter signal on

605 height measurements. However this overestimation is lower than the standard deviation of the terrain measurements indicating a good accuracy of estimates.

The accuracy of the method used for estimating top height should depend on stand characteristics. Stands with fewer trees would normally require a greater number

610 of measurements for computing an accurate top height. The less accurate estimation of top height in the young plantation could possibly be linked to a bias in the reference data. Only one out of every three trees was measured in the young stand plots. A detailed inspection of the reference data collected in plots y1 and plot y2 showed that the total height of the 9 trees having the largest diameters at breast height in each plot

615 were not measured. Consequently, the reference top height was not accurately set in the young plantation, resulting in a gap with the lidar-retrieved top height. The underestimation of the top height in the mature stand is certainly linked to the causes of underestimation already discussed above. However, the reliability of the top height assessment in the mature stand was demonstrated, as no error exists in reference data, and the bias was lower than the standard deviation of the terrain measurements.

The slight difference between measurements computed for the entire dataset and for only one flight line shows that a $2.4 \mathrm{~m}$ wide non-continuous transect across an homogeneous stand allows to provide reliable mean tree height, mean crown height and top height estimates. This result is highly encouraging for providing forest inventories on large area with low costs. If parcel or of stand-type maps are available, a well designed flightplan with at least one flight line intersecting each forest unit would provide mean and top heights at least on even-aged stands. Other experiments are required to check if this result is still valid on multilayered forests, but we can imagine

630 that one flight line over randomly positioned trees will give a good description of the entire stand.

\subsection{Phase 3: Planting pattern and tree spacing assessment}

635 Contrary to height retrieval methods, the following analysis using variograms needs an explicit lidar sampling of the forest stand to work. At least, it would require two perpendicular flight lines to compute directional variograms. Several flight lines in two perpendicular direction would provide an even bigger amount of measurement pairs per class of distance for computing significant variograms. In this study, flight lines 
640 where performed in only one direction. Consequently, we got a higher number of measurement pairs in the direction of the flight lines. However, the ULA attitude provided not always straight measurements, allowing then to get a sufficient number of pairs in every direction. We checked that each variogram sample was computed with a minimum of 80 distinct pairs.

In fig. 5, we can notice a weak behavior in semi-variance between $6 \mathrm{~m}$ and $8 \mathrm{~m}$ for directions $0^{\circ}$ and $30^{\circ}$ (a decrease followed by an increase in semi-variance) resulting from some measurement variations that were not totally captured at small distances. Soil physical properties may produce such a cyclic phenomenon in variograms also

650 known as "hole-effect" (Journel and Huijbregts, 1978). Soil samples have to be taken to validate this hypothesis, but the aim of the directional variogram analysis was only to assess the presence of an anisotropy based on the nugget parameter.

Associating a confidence value to the detection of a directional anisotropy would have been useful, but it was not possible to perform due to the difficulty in simulating a non-

655 Gaussian distribution of height for such a test. The way to calculate a confidence value is to verify whether an empirical directional variogram is included in the confidence envelope built from a simulated data set satisfying an isotropy hypothesis (Lantuejoul 2002). The simulated data set also needs to fit the model previously adjusted on the empirical omnidirectional variogram, and this methodology requires a Gaussian

660 distribution of the studied variable. Because the distribution of our lidar data was nonGaussian, as it was not composed of individual trees heights but of maximum heights measured within footprints, such a test needs further methodological development. Such developments, at present time, should rely on transforming non-Gaussian to Gaussian fields or indicator fields (Emery, 2002).

The variogram model is a continuous function defined in $\mathbb{R}^{+}$. Although variogram estimation starts at distance $1.2 \mathrm{~m}$ (Fig. 6) due to the minimum spacing between two lidar measurements, the function was plotted from distance $0 \mathrm{~m}$ where it always have variance 0 . The variogram function values between 0 and $1.2 \mathrm{~m}$ result from

670 model choice and parameters fit. They mainly reveal the nugget parameter with an uncertainty due to the lack of data at these distances. However, the method presented in this paper allows estimating the mean spacing between trees through the practical range parameter. The uncertainty between 0 and $1.2 \mathrm{~m}$ did not affect the practical range parameter and the tree spacing was accurately estimated on the semi-natural stand. In

675 the young stand, the mean spacing between trees in a line and between lines may differ, and they could not be extracted from the unique omnidirectional variogram model. Nevertheless, it may be possible to model directional variograms to retrieve tree spacing in specific directions. In this study, however, the georeferencing accuracy was insufficient to finely estimate the metrics using modeled directional variograms on different series of 20 shoots. A shoot was well georeferenced relative to the others 
inside the same group of 20 shoots, but as explained in section 2.2, the absolute accuracy (between two series of shoots) was about $4.5 \mathrm{~m}$. Such accuracy was insufficient to extract metrics from directional variograms in the perpendicular direction of the flight lines, but we demonstrated the possibility of identifying tendencies in the spatial distribution of trees, such as the identification of the planting pattern.

Despite that the variogram based methods cannot be used to process a one flight line profiler dataset, it could be of great interest to process small-footprint lidar data. It would allow retrieving mean inter-tree distance, and therefore stand density, through a

690 direct processing of the first return lidar point cloud after correcting heights from ground elevation, instead of searching for local maxima on previously interpolated raster Digital Canopy Models.

\subsection{Spaceborne UV lidar}

695

Studying vegetation and aerosols at the same time was not possible in this experiment because the sensor was too close to the forest. Aerosols detection would have required the study of too small signal variations compared to those produced by the laser backscattering on the canopy. But a simultaneous detection of both forest and

700 aerosols would be easier from satellite platforms due to a lower signal dynamics caused by the atmospheric transmission coefficient.

Beside Rayleigh scattering caused by atmosphere gases, clouds will play a major role in laser attenuation. Despite that semi-transparent cirrus clouds will not have a drastic impact on laser move forward, dense cumulus-type clouds will produce signal

705 extinction. But the problem is not limited to ultraviolet wavelength and also occurs in both visible and near-infrared wavelenghts (Chazette et al., 2001). However, the UV laser would be less sensitive to multiple backscattering in the vegetation, due to a smaller leaf reflectance in the UV compared to NIR (Grant et al., 2003), and would consequently increase the accuracy of ground positioning under the canopy compared to

710 existing spaceborne lidars.

\section{Conclusion}

This paper presents methods developed for retrieving forest parameters from a

715 new, eye-safe medium-footprint lidar sensor initially designed for atmospheric applications using an ultraviolet laser. The advantage of a medium-footprint size is that it provides higher resolution data than large footprint systems while increasing the probability of reaching tree tops more effectively than small-footprint lidar data. A first evaluation of the system and of its potential for forest parameter retrieval took place in

720 one of the major planted forests in Europe, the Landes forest in France. Both the simple stand structure and the flat topography were expected to make the data processing and 
interpretation of results easier.

The methods presented in this paper were developed to retrieve mean total height, mean crown height, top height, planting pattern and tree spacing according to

725 the specificity of medium-footprint lidar data. Despite the absolute positioning uncertainties due to the lack of ULA accurate attitude data, the mean total height, mean crown height and top height were estimated with an absolute error generally lower than the standard deviation of the reference measurements. In addition, the obtained results regarding crown height estimation were better than in the previous study (Cuesta et al.,

730 2010), demonstrating the importance of correcting the signal from its attenuation for assessing forest structure inside the canopy. A method using geostatistics for recognizing the planting pattern was also performed successfully, and the tree spacing was accurately estimated for the semi-natural stand. Although the methods presented in this paper were developed for medium-footprint lidar data, they can be also used for

735 processing small- or large-footprint lidar data. In particular, the correction of laser attenuation inside vegetation is applicable to any kind of lidar waveform, while the geostatistically based processes might give even more precise results on a smallfootprint lidar dataset which is normally of better absolute precision than the one available here.

740 Consequently, the LAUVA prototype demonstrated its ability to assess forest structure. The lightness of this lidar sensor allows the use of an ultra-light aircraft, which can be more rapidly deployed than other airborne missions. The cost is also lower, particularly for surveys of small areas, which are not optimal with other airborne platforms. In addition, we demonstrated that measuring canopy height along a single

$7452.4 \mathrm{~m}$ wide transect across a stand is sufficient to provide reliable usual inventory parameters, which can be of great interest to perform larger scale inventories at a minimal cost (saving flight time, data storage and process). It is therefore a valuable sensor for performing emergency forest inventory. However, we hope to increase the georeferencing accuracy using a finer IMU in future developments. It would allow us to

750 accurately position each laser spot in a global referential. When a dense and spatially explicit sampling strategy is chosen this would make possible the computation of high resolution digital terrain models, and would allow further investigations on variogram methods to refine the estimation of forest parameters. The waveform digitization frequency will also be increased, providing additional accuracy for tree height

755 measurements. As LAUVA is a profiler system, the sampling strategy could be rethought on the basis of the results obtained in this study to optimize the sampling strategy and increase the speed of data acquisition. One option would be to perform continuous measurements with only one transect per parcel. In such a configuration, the processing methods will also need to be rethought and will probably be closer to

760 existing process for spaceborne lidar. The success in forest structure estimation also gives confidence for the adaptation of this system for a spaceborne mission (Flamant, 2005) with a smaller footprint than existing systems. To that aim, additional 
experiments will be performed at different flight altitudes, for obtaining various footprint sizes up to 10-15 $\mathrm{m}$ ? Hence, the impact of the footprint dimension on

765 measurement accuracy of forest structure will be assessed. The main advantage of such a system would be to limit the terrain slope influence on the tree height measurements compared to ICESat system with a $70 \mathrm{~m}$ footprint diameter. However, this system will require further methodological work to process waveforms with footprints smaller than $72 \mathrm{~m}^{2}$. Finally, as the initial function of the LAUVA system was to measure

770 atmospheric aerosols (Raut and Chazette, 2009), the first results obtained on forests confirm that the conception of a bi-functional lidar for studying forest responses to atmospheric pollution seems also feasible.

\section{Acknowledgments}

This work is part of the ExFOLIO project and was realized thanks to the financial support of the CNES (Centre National d'Études Spatiales), the CEA, the CNRS (PNTS funding), and the French Languedoc-Roussillon region. The authors also thank the Mimizan town hall representatives (Mrs. Dehureau-Foidart and Mr. Bertrand)

780 for facilitating the mission. We also thank Laurent Albrech, from UMR TETISCemagref, for developing the georeferencing algorithm.

\section{References}

785 Boone, E. L., Bullock, B. P., 2008. Spatial correlation matrix selection using Bayesian model averaging to characterize inter-tree competition in loblolly pine trees. Journal of Applied Statistics 35 (9), 967-977.

Brandtberg, T., Warner, T. A., Landenberger, R. E., McGraw, J. B., 2003. Detection

790 and analysis of individual leaf-off tree crowns in small footprint, high sampling density lidar data from the eastern deciduous forest in North America, Remote Sensing of Environment 85 (3), 290-303.

Chauve, A.,Vega, C., Durrieu, S., Bretar, S., Allouis, T., Pierrot-Deseilligny, M., Puech, 795 W., 2009. Advanced fullwaveform lidar data echo detection: Assessing quality of derived terrain and tree height models in an alpine coniferous forest. International Journal of Remote Sensing 30 (19), 5211-5228.

Chazette, P., Pelon, J., Mégie, G., 2001. Determination by spaceborne backscatter lidar 800 of the structural parameters of atmospheric scattering layers, Applied Optics 40 (21), 3428-3440.

Chazette, P., Sanak, J., Dulac, F., 2007. New approach for aerosol profiling with a lidar 
onboard an ultralight aircraft: application to the African monsoon. Environmental

Chazette, P., Raut, J.-C., Dulac, F., Berthier, S., Kim, S-W., Royer, P., Sanak, J., Loaëc, S., Grigaut-Desbrosses, H., 2010. Simultaneous observations of lower tropospheric continental aerosols with a ground-based, an airborne, and the spaceborne CALIOP

810 lidar systems, Journal of Geophysical Research 115 (16), D00H31.

Chen, Q., Gong, P., Baldocchi, D., Tian, Y. Q., 2007. Estimating basal area and stem volume for individual trees from lidar data. Photogrammetric Engineering and Remote Sensing 73 (12), 1355-1365.

Chen, Q., 2010. Retrieving vegetation height of forests and woodlands over mountainous areas in the Pacific Coast region using satellite laser altimetry. Remote Sensing of Environment 114 (7), 1610-1627.

820 Chiles, J. P., Delfiner, P., 1999. Geostatististics: Modelling spatial uncertainty, John Wiley \& Sons Inc., New York.

Clark, M. L., Clark, D. B., Roberts, D. A., 2004. Small-footprint lidar estimation of subcanopy elevation and tree height in a tropical rain forest landscape, Remote Sensing of

825 Environment 91 (1), 68-89.

Cressie, N., 1993. Statistics for spatial data, Wiley Interscience.

Cuesta, J., Chazette, P., Allouis, T., Flamant, P. H., Durrieu, S., Sanak, J., Genau, P., 830 Guyon, D., Loustau, D., Flamant, C., 2010. Observing the Forest Canopy with a New Ultra-Violet Compact Airborne Lidar, Sensors 10 (8), 7386-7403.

Curran, P. J., 1988. The semivariogram in remote sensing: An introduction, Remote Sensing of Environment 24 (3), 493-507.

DeFries, R., Achard, F., Brown, S., Herold, M., Murdiyarso, D., Schlamadinger, B., de Souza Jr., C., 2007. Earth observations for estimating greenhouse gas emissions from deforestation in developing countries. Environmental Science \& Policy 10 (4), 385-394.

840 Delenne, C., Durrieu, S., Rabatel, G., Deshayes, M., Bailly, J. S., Lelong, C. Couteron, P., 2008. Textural approaches for vineyard detection and characterization using very high spatial resolution remote sensing data. International Journal of Remote Sensing 29 (4), 1153-1167. 
Author-produced version of the article published in ISPRS Journal of Photogrammetry and Remote Sensing, 2011, 66(6), S92-S102.

845 Drake, J. B., Dubayah, R. O., Clark, D. B., Knox, R. G.k, Blair, J. B., Hofton, M. A., Chazdon, R. L., Weishampel, J. F., Prince, S., 2002. Estimation of tropical forest structural characteristics, using large-footprint lidar, Remote Sensing of Environment 79 (2-3), 305-319.

850 Emery, X., 2002. Conditional simulation of nongaussian random functions. Mathematical Geology 34 (1), 79-100.

ESA, 2008. Biomass, Mission assessment report, SP-1313/2. http://esamultimedia.esa.int/docs/SP1313-2_BIOMASS.pdf (Accessed 4 July, 2011)

Flamant, P.H., 2005. Atmospheric and meteorological Lidar: from pioneers to space applications. Comptes Rendus Physique 6 (8), 864-875.

Garcia, O., Batho, A., 2005. Top Height Estimation in Lodgepole Pine Sample Plots, 860 Western Journal of Applied Forestry 20 (1), 64-68.

Gille, W., 2002. The Set Covariance of a Dead Leaves Model, Advances in Applied Probability 34 (1), 11-20.

865 Grant, R. H., Heisler, G. M., Gao, W., Jenks, M., 2003. Ultraviolet leaf reflectance of common urban trees and the prediction of reflectance from leaf surface characteristics, Agricultural and Forest Meteorology 120 (1-4), 127-139.

Harding, D. J., Carabajal, C. C., 2005. ICESat waveform measurements of within870 footprint topographic relief and vegetation vertical structure. Geophysical Research Letters 32 (21), 1-4.

Hofton, M. A., Minster, J. B., Blair, J. B., 2000. Decomposition of laser altimeter waveforms, IEEE Transactions on Geoscience and Remote Sensing 38 (4 II), 19898751996.

Journel, A. G., Huijbregts, C. J., 1978. Mining Geostatistics. Academic Press, New York.

880 Kato, A., Moskal, L. M., Schiess, P., Swanson, M. E., Calhoun, D., Stuetzle, W., 2009. Capturing tree crown formation through implicit surface reconstruction using airborne lidar data, Remote Sensing of Environment 113 (6), 1148-1162.

Lantuejoul, C., 2002. Geostatistical Simulation: Models and Algorithms, Springer 885 Verlag, Berlin, pp. 256. 
Lefsky, M. A., Cohen, W. B., Acker, S. A., Parker, G. G., Spies, T. A., Harding, D., 1999. Lidar remote sensing of the canopy structure and biophysical properties of Douglas-fir western hemlock forests, Remote Sensing of Environment 70 (3), 339-361.

Lefsky, M. A., Harding, D. J., Keller, M., Cohen, W. B., Carabajal, C. C., Del Bom Espirito-Santo, F., Hunter, M. O., de Oliveira Jr., R., 2005. Estimates of forest canopy height and aboveground biomass using ICESAT. Geophysical Research Letters 32 (22), $1-4$.

MacArthur, R. H. \& Horn, H. S., 1969. Foliage Profile by Vertical Measurements, Ecology 50 (5), 802-804.

Mallet, C., Bretar, F., 2009. Full-waveform topographic lidar: State-of-the-art, ISPRS 900 Journal of Photogrammetry and Remote Sensing 64 (1), 1-16.

Means, J. E., Acker, S. A., Harding, D. J., Blair, J. B., Lefsky, M. A., Cohen, W. B., Harmon, M. E., McKee, W. A., 1999. Use of large-footprint scanning airborne Lidar to estimate forest stand characteristics in the western cascades of Oregon. Remote Sensing 905 of Environment 67 (3), 298-308.

Means, J. E., Acker, S. A., Fitt, B. J., Renslow, M., Emerson, L., Hendrix, C. J., 2000. Predicting forest stand characteristics with airborne scanning lidar. Photogrammetric Engineering and Remote Sensing 66 (11), 1367-1371.

Measures, R. M., 1984. Laser remote sensing: Fundamentals and Applications. John Wiley \& Sons Inc, NewYork.

Næsset, E., 2009. Effects of different sensors, flying altitudes, and pulse repetition 915 frequencies on forest canopy metrics and biophysical stand properties derived from small-footprint airborne laser data. Remote Sensing of Environment 113 (1), 148-159.

Nakai, T., Sumida, A., Kodama, Y., Hara, T., Ohta, T., 2010. A comparison between various definitions of forest stand height and aerodynamic canopy height, Agricultural 920 and Forest Meteorology 150 (9), 1225-1233.

Packalen, P., Suvanto, A., Maltamo, M., 2009. A two stage method to estimate speciesspecific growing stock. Photogrammetric Engineering and Remote Sensing 75 (12), 1451-1460.

Popescu, S. C., 2007. Estimating biomass of individual pine trees using airborne lidar, 
Author-produced version of the article published in ISPRS Journal of Photogrammetry and Remote Sensing, 2011, 66(6), S92-S102. The original publication is available at http://www.isprs.org/

DOI:10.1016/j.isprsjprs.2011.08.007

Biomass and Bioenergy 31 (9), 646-655.

Ranchin, T., Naert, B., Albuisson, M., Boyer, G., Astrand, P., 2001. An automatic

930 method for vine detection in airborne imagery using wavelet transform and multiresolution analysis. Photogrammetric Engineering and Remote Sensing 67 (1), 9198.

Raut J.-C., Chazette, P., 2009. Assessment of vertically-resolved PM10 from mobile 935 lidar observations. Atmospheric Chemistry and Physics 9 (21), 8617-8638.

Ribeiro Jr, P. J., Diggle, P. J., 2010. Package geoR version 1.6-27.

Silva-Santos, P., Valentim, H., Luís, A., Queirós, L., Travassos, P., Cabral, J. A., 2010.

940 Stochastic Dynamic Methodology (StDM) to simulate the effects of fire on vegetation and bird communities in Pinus pinaster stands. Ecological Indicators 10 (2), 206-211.

Soenen, S. A., Peddle, D. R., Hall, R. J., Coburn, C. A., Hall, F. G., 2010. Estimating aboveground forest biomass from canopy reflectance model inversion in mountainous

945 terrain. Remote Sensing of Environment 114 (7), 1325-1337.

St-Onge, B. A., Cavayas, F., 1997. Automated forest structure mapping from high resolution imagery based on directional semivariogram estimates. Remote Sensing of Environment 61 (1), 82-95.

Stam, D. M., 2008. Spectropolarimetric signatures of Earth-like extrasolar planets, Astronomy and Astrophysics 482 (3), 989-1007.

Tesfamichael, S., Ahmed, F., van Aardt, J., Blakeway, F., 2009. A semi-variogram 955 approach for estimating stems per hectare in Eucalyptus grandis plantations using discrete-return lidar height data. Forest Ecology and Management 258 (7), 1188-1199.

Véga, C., St-Onge, B., 2008. Height growth reconstruction of a boreal forest canopy over a period of 58 years using a combination of photogrammetric and lidar models.

960 Remote Sensing of Environment 112 (4), 1784-1794.

Wackernagel, H., 1995. Multivariate Geostatistics, Springer Verlag, Berlin.

Waser, L., Baltsavias, E., Ecker, K., Eisenbeiss, H., Feldmeyer-Christe, E., Ginzler, C., 965 Küchler, M., Zhang, L., 2008. Assessing changes of forest area and shrub encroachment in a mire ecosystem using digital surface models and CIR aerial images. Remote Sensing of Environment 112 (5), 1956 - 1968. 
Author-produced version of the article published in ISPRS Journal of Photogrammetry and Remote Sensing, 2011, 66(6), S92-S102. The original publication is available at http://www.isprs.org/

DOI:10.1016/j.isprsjprs.2011.08.007

Williston, H.L., Balmer, W.E., 1974. Managing for Natural Regeneration. USDA Forest 970 Service State and Private Forestry. Forest Management Bulletin. 6 p.

Wynne, R. H., 2006. Lidar remote sensing of forest resources at the scale of management. Photogrammetric Engineering and Remote Sensing 72 (12), 1310-1314.

975 Zimmerman, D. L., 1993. Another look at anisotropy in geostatistics. Mathematical Geology 25 (4), 453-470. 